\title{
MIMO-OFDM Training Signal Design in the Presence of Frequency Offset and Phase Noise
}

\author{
Hlaing Minn*, Member, IEEE and Naofal Al-Dhahir, Senior Member, IEEE \\ University of Texas at Dallas, Richardson, TX 75083-0688, U.S.A. \\ Email: hlaing.minn@utdallas.edu, aldhahir@utdallas.edu
}

\begin{abstract}
All existing training signal designs for channel estimation in OFDM systems neglect phase noise or both frequency offsets and phase noise. In practice, frequency offset and phase noise are unavoidable and can seriously degrade the performance of OFDM systems. In this paper, we address the problem of designing optimal training signals for the estimation of frequencyselective channels in MIMO OFDM systems with frequency offset and phase noise. Analytical and simulation results for the corresponding channel estimation mean square error are presented. Channel estimation performance is more sensitive to frequency offset and phase noise in MIMO than in SISO OFDM systems. The performance improvement achieved by the proposed training signals becomes more significant for moderate-to-high values of SNR, frequency offset, and phase noise level.
\end{abstract}

\section{INTRODUCTION}

All existing OFDM-based systems transmit training signals for synchronization and channel estimation purpose. Since a proper training signal design can improve estimation performance and reduce the estimation complexity substantially, it has attracted significant research attention. Training signal designs for synchronization were addressed in [1]-[3]. For channel estimation, [4]-[7] presented training signal designs for single-input single-output (SISO) OFDM systems while [8]-[10] addressed training designs for multi-input multioutput (MIMO) OFDM systems. Recently in [11], using basic properties of the discrete Fourier transform, we presented general optimal training signal designs for frequency-selective fading channel estimation in MIMO OFDM systems. The optimal pilot tone allocations are classified as frequency-division multiplexing (FDM), time-division multiplexing (TDM), codedivision multiplexing in frequency-domain (CDM(F)), codedivision multiplexing in time-domain $(\mathrm{CDM}(\mathrm{T}))$ and combinations thereof. These classifications include all existing optimal training signal designs for MIMO OFDM fading channel estimation in the literature as special cases.

All of the above existing training signal designs for OFDM channel estimation assume no frequency offset and phase noise. In practice, frequency offset is unavoidable due to local oscillator mismatches. Due to non-ideal oscillators, phase noise is unavoidable too. Frequency offset and phase noise cause a loss of orthogonality among the sub-carriers which in turn can seriously degrade the performance of OFDM systems [13]. Recently, we have presented training signal designs for MIMO-OFDM channel estimation in the presence of frequency offsets in [12] where it is shown that the channel estimation performance can be severely degraded by frequency offsets and our training signal designs can alleviate the degradation significantly.

In this paper, we present training signal designs for MIMOOFDM channel estimation in the presence of both frequency offset and phase noise. Our results show that MIMO OFDM systems are more sensitive to frequency offsets and phase noise than SISO OFDM systems and the performance improvement of the proposed optimal training signals becomes more significant for moderate-to-high values of SNR, frequency offset, and phase noise level.

The rest of this paper is organized as follows. Section II describes the signal model. Section III presents the proposed design. Simulation results and discussions are presented in Section IV and the paper is concluded in Section V.

\section{SignAL MODEL}

Consider a MIMO OFDM system where training signals from $N_{\mathrm{Tx}}$ transmit antennas are transmitted over $Q$ OFDM symbols. Since the same channel estimation procedure is performed at each receive antenna, we only need to consider one receive antenna in designing training signals. The channel impulse response (CIR) for each transmit-receive antenna pair (including all transmit/receive filtering effects) is assumed to have $L$ taps and is quasi-static over $Q$ OFDM symbols. Let $C_{n, q}=\left[c_{n, q}[0], \ldots, c_{n, q}[K-1]\right]^{T}$ be the pilot tones vector of the $n$-th transmit-antenna at the $q$-th symbol interval where $K$ is the number of OFDM sub-carriers and the superscript $T$ denotes the transpose. Furthermore, let $\left\{s_{n, q}[k]: k=\right.$ $\left.-N_{g}, \ldots, K-1\right\}$ be the corresponding time-domain complex baseband training samples, including $N_{g}(\geq L-1)$ cyclic prefix samples. Define $\boldsymbol{S}_{n}[q]$ as the training signal matrix of size $K \times L$ for the $n$-th transmit antenna at the $q$-th symbol interval whose elements are given by $\left[\boldsymbol{S}_{n}[q]\right]_{m, l}=s_{n, q}[m-l]$ for $m \in\{0, \ldots, K-1\}$ and $l \in\{0, \ldots, L-1\}$.

Define $s_{n, q}$ as the 0 -th column of $\boldsymbol{S}_{n}[q]$. Then, the $l$-th column of $\boldsymbol{S}_{n}[q]$ is the $l$-sample cyclically-shifted version of $s_{n, q}$ denoted by $s_{n, q}^{((l))}$. Let $h_{n}$ denote the length- $L$ CIR vector corresponding to the $n$-th transmit antenna. After cyclic prefix removal at the receiver, denote the received vector of length $K$ at the $q$-th symbol interval by $\boldsymbol{r}_{q}$. Then, the received vector over the $Q$ symbol intervals in the absence of frequency offset and phase noise is given by

$$
r=S h+n
$$

where

$$
\boldsymbol{r}=\left[\begin{array}{llll}
\boldsymbol{r}_{0}^{T} & \boldsymbol{r}_{1}^{T} & \ldots & \boldsymbol{r}_{Q-1}^{T}
\end{array}\right]^{T}
$$




$$
\begin{aligned}
\boldsymbol{h}= & {\left[\boldsymbol{h}_{0}^{T} \boldsymbol{h}_{1}^{T} \ldots \boldsymbol{h}_{N_{\mathrm{Tx}}-1}^{T}\right]^{T} } \\
\boldsymbol{S}= & {\left[\begin{array}{llll}
\boldsymbol{S}_{0}[0] & \boldsymbol{S}_{1}[0] & \ldots & \boldsymbol{S}_{N_{\mathrm{Tx}}-1}[0] \\
\boldsymbol{S}_{0}[1] & \boldsymbol{S}_{1}[1] & \ldots & \boldsymbol{S}_{N_{\mathrm{Tx}}-1}[1] \\
\vdots & \vdots & \ddots & \vdots \\
\boldsymbol{S}_{0}[Q-1] & \boldsymbol{S}_{1}[Q-1] & \ldots & \boldsymbol{S}_{N_{\mathrm{Tx}}-1}[Q-1]
\end{array}\right] }
\end{aligned}
$$

and $\boldsymbol{n}$ is a length- $K Q$ vector of circularly-symmetric, independent and identically-distributed (iid) complex Gaussian noise samples with zero-mean and variance of $\sigma_{n}^{2}$.

The least-squares channel estimate (also maximum likelihood in this case), assuming $S^{H} S$ is full rank, is given by

$$
\hat{h}=\left(S^{H} S\right)^{-1} S^{H} r
$$

and the corresponding mean square error (MSE) is given by

$$
\mathrm{MSE}=\sigma_{n}^{2} \operatorname{Tr}\left\{\left(\boldsymbol{S}^{H} \boldsymbol{S}\right)^{-1}\right\} .
$$

The training signal designs from [8]-[11] minimize the above MSE. The minimum MSE is achieved if and only if

$$
\begin{aligned}
\boldsymbol{S}^{H} \boldsymbol{S} & =E_{\mathrm{av}} \boldsymbol{I} \\
\text { where } \quad E_{\mathrm{av}} & =\frac{1}{N_{\mathrm{Tx}}} \sum_{n=0}^{N_{\mathrm{Tx}}-1} E_{n} \\
\text { and } \quad E_{n} & =\sum_{q=0}^{Q-1} \sum_{k=0}^{K-1}\left|s_{n, q}[k]\right|^{2} .
\end{aligned}
$$

The corresponding minimum MSE is $L N_{\mathrm{Tx}} \sigma_{n}^{2} / E_{\mathrm{av}}$.

In the presence of a normalized carrier frequency offset $v$, the received vector in (1) becomes

$$
r=\boldsymbol{W}(v) \boldsymbol{S h}+\boldsymbol{n}
$$

where $\boldsymbol{W}(v)=\operatorname{diag}\left\{\boldsymbol{W}_{0}(v), e^{\frac{j 2 \pi v\left(K+N_{g}\right)}{K}} \boldsymbol{W}_{0}(v)\right.$, $\left.e^{\frac{j 2 \pi v 2\left(K+N_{g}\right)}{K}} \boldsymbol{W}_{0}(v), \quad \ldots \quad, e^{\frac{j 2 \pi v(Q-1)\left(K+N_{g}\right)}{K}} \boldsymbol{W}_{0}(v)\right\} \quad$ and $\boldsymbol{W}_{0}(v)=\operatorname{diag}\left\{1, e^{\frac{j 2 \pi v}{K}}, e^{\frac{j 2 \pi 2 v}{K}}, \ldots, e^{\frac{j 2 \pi(K-1) v}{K}}\right\}$. From $(5),(7)$, and (10), the corresponding channel estimate can be expressed as

$$
\begin{aligned}
\hat{h}=\frac{1}{E_{\mathrm{aV}}} \boldsymbol{S}^{H} r & =h-\frac{1}{E_{\mathrm{aV}}}\left\{\boldsymbol{S}^{H}(\boldsymbol{I}-\boldsymbol{W}(v)) \boldsymbol{S} h-\boldsymbol{S}^{H} \boldsymbol{n}\right\} \\
& \equiv h-\Delta_{h} .
\end{aligned}
$$

Define the normalized MSE as

$$
\begin{aligned}
& \mathrm{NMSE}=\frac{\mathrm{MSE}}{L N_{\mathrm{Tx}}} \\
& =\frac{\sigma_{n}^{2}}{E_{\mathrm{av}}}+\frac{\operatorname{Tr}\left[\boldsymbol{S}^{H}(\boldsymbol{I}-\boldsymbol{W}(v)) \boldsymbol{S} \boldsymbol{C}_{h} \boldsymbol{S}^{H}(\boldsymbol{I}-\boldsymbol{W}(v))^{H} \boldsymbol{S}\right]_{(12}}{L N_{\mathrm{Tx}} E_{\mathrm{av}}^{2}} \\
& \equiv \mathrm{NMSE}_{0}+\Delta_{\mathrm{NMSE}}
\end{aligned}
$$

where the first term is the NMSE obtained without any frequency offset or phase noise, the second term is the extra NMSE caused by the frequency offset $v$, and $C_{h}$ is the covariance matrix of the CIR vector $h$ from (3). Since NMSE 0 is the same for all training signals which were optimal in the absence of frequency offset and phase noise, the training signal designs from [12] minimizes $\Delta_{\mathrm{NMSE}}$.

In addition to frequency offset, there also exists phase noise in practical systems. The signal model in the presence of frequency offset and phase noise is obtained by replacing $\boldsymbol{W}(v)$ in (10) with $\overline{\boldsymbol{W}}(v)$ which is given by

$$
\overline{\boldsymbol{W}}(v)=\boldsymbol{W}(v) \boldsymbol{\Phi}
$$

where

$$
\begin{aligned}
\Phi= & \operatorname{diag}\left\{e^{j \phi_{0}}, \ldots, e^{j \phi_{K-1}}, e^{j \phi_{K+N_{g}}}, \ldots, e^{j \phi_{2 K+N_{g}-1}},\right. \\
& \left.\ldots, e^{j \phi_{(Q-1)\left(K+N_{g}\right)}}, \ldots, e^{j \phi_{(Q-1)\left(K+N_{g}\right)+K-1}}\right\}
\end{aligned}
$$

and $\left\{\phi_{k}\right\}$ are the phase-noise samples. A continuous-time phase noise $\phi(t)$ is commonly modeled by a Wiener process with $E[\phi(t)]=0$ and $E\left[(\phi(t+\tau)-\phi(t))^{2}\right]=4 \pi \beta|\tau|$ where $\beta$ denotes the one-sided $3 \mathrm{~dB}$ linewidth of the Lorentzian power density spectrum of the oscillator [13]. In the discrete-time domain, the phase noise samples can be modeled by $\phi_{n}=$ $\phi_{n-1}+\Delta \phi$ where $\Delta \phi$ is a sample of white Gaussian process with variance $\sigma_{p}^{2}$ and $\phi_{0}$ is uniformly distributed over $[-\pi, \pi)$. Note that $\sigma_{p}^{2}=4 \pi \beta /\left(K \Delta_{f}\right)$ where $\Delta_{f}$ is the sub-carrier spacing. Since $\phi_{0}$ can be embedded in the CIR vector, it can simply be set to zero.

In the presence of frequency offset and phase noise, the NMSE is given by

$$
\begin{aligned}
& \text { NMSE } \\
& =\frac{\sigma_{n}^{2}}{E_{\mathrm{av}}}+\frac{E\left[\operatorname{Tr}\left[\boldsymbol{S}^{H}(\boldsymbol{I}-\overline{\boldsymbol{W}}(v)) \boldsymbol{S} C_{h} \boldsymbol{S}^{H}(\boldsymbol{I}-\overline{\boldsymbol{W}}(v))^{H} \boldsymbol{S}\right]\right]}{L N_{\mathrm{Tx}} E_{\mathrm{av}}^{2}} \\
& \equiv \mathrm{NMSE}_{0}+\Delta_{\mathrm{NMSE}}
\end{aligned}
$$

where the first term is the NMSE obtained in the absence of frequency offset and phase noise and the second term is the extra NMSE caused by the frequency offset and phase noise. Then our training signal design for MIMO-OFDM channel estimation becomes minimizing $\Delta_{\mathrm{NMSE}}$ from (16).

\section{TRAINING SIGNAL DESIGN IN THE PRESENCE OF FREQUENCY OFFSET AND PHASE NOISE}

Following the same approach from [12], we just need to consider the training signals defined by

$$
\begin{aligned}
s_{k, q}[n] & =\sum_{i=0}^{d_{k, q}-1} A_{k, q, i} \delta\left[n-l_{k, q, i}\right], k=0, \ldots, N_{\mathrm{Tx}}-1(17) \\
d_{q} & \equiv \sum_{k=0}^{N_{\mathrm{Tx}}-1} d_{k, q} \leq \frac{N}{L}
\end{aligned}
$$

where $d_{k, q}$ is the number of non-zero samples of the $q$-th OFDM training symbol (excluding CP samples) for the $k$ th transmit antenna and for each $q,\left\{l_{k, q, i}: \forall k, i\right\}$ are any permutation of $\left\{m_{p}\right\}$ with $m_{p+1}-m_{p} \geq L, K+m_{0}-$ $m_{d_{q}-1} \geq L$, and $0 \leq m_{p} \leq K-1$. In addition, each transmit antenna must have the same total transmitted training energy [11] which, in turn, implies that

$$
\sum_{q=0}^{Q-1} \sum_{i=0}^{d_{k, q}-1}\left|A_{k, q, i}\right|^{2}=E_{\mathrm{av}}, \forall k .
$$

The results from [12] show that under the same total training signal energy constraint, using one OFDM training symbol (i.e., $Q=1$ ) is more robust to frequency offsets than using multiple OFDM training symbols. Hence, for simplicity, we will consider one OFDM training symbol (i.e., $Q=1$ ) and the symbol index $q$ will be omitted.

Then our training signal design that minimizes $\Delta_{\mathrm{NMSE}}$ from (16) becomes

$$
\left\{A_{k, i}, l_{k, i}, d_{k}\right\}^{\star}=\underset{\left\{A_{k, i}, l_{k, i}, d_{k}\right\}}{\arg \min } \operatorname{Tr}\left[\overline{\boldsymbol{X}}_{d}\right]
$$


where the optimization constraints are given by (17)-(19) and associated conditions, and $\operatorname{Tr}\left[\overline{\boldsymbol{X}}_{d}\right]$ is given by

$$
\begin{array}{r}
\operatorname{Tr}\left[\overline{\boldsymbol{X}}_{d}\right] \equiv \operatorname{Tr}\left[E\left[\boldsymbol{S}^{H}(\boldsymbol{I}-\overline{\boldsymbol{W}}(v)) \boldsymbol{S} \boldsymbol{C}_{h} \boldsymbol{S}^{H}(\boldsymbol{I}-\overline{\boldsymbol{W}}(v))^{H} \boldsymbol{S}\right]\right] \\
=\sum_{k=0}^{N_{\mathrm{Tx}}-1} \sum_{m=0}^{L-1} \sigma_{m}^{2} E\left[\left.\left.\left|\sum_{i=0}^{d_{k}-1}\right| A_{k, i}\right|^{2} \bar{V}_{m+l_{k, i}}\right|^{2}\right] \\
=\sum_{k=0}^{N_{\mathrm{Tx}}-1} \sum_{m=0}^{L-1} \sigma_{m}^{2}\left\{\sum_{i=0}^{d_{k}-1}\left|A_{k, i}\right|^{4} E\left[\left|\bar{V}_{m+l_{k, i}}\right|^{2}\right]\right. \\
\left.+2 \sum_{i=0}^{d_{k}-1} \sum_{n=1_{i}}^{d_{k}-1}\left|A_{k, i}\right|^{2}\left|A_{k, n}\right|^{2} \Re\left[E\left[\bar{V}_{m+l_{k, i}} \bar{V}_{m+l_{k, n}}^{*}\right]\right]\right\}
\end{array}
$$

where $\bar{V}_{l}$ is $l$-th diagonal element of $\overline{\boldsymbol{V}}(v) \equiv(\boldsymbol{I}-\overline{\boldsymbol{W}}(v))$ and the correlation of $\left\{\bar{V}_{l}\right\}$ is obtained by a straight-forward calculation as

$$
\begin{aligned}
& E\left[\bar{l}_{l} \bar{V}_{m}^{*}\right]=E\left[\left(1-e^{j\left(\phi_{k_{l}}+\frac{2 \pi k_{l} v}{K}\right)}\right)\left(1-e^{-j\left(\phi_{k_{m}}+\frac{2 \pi k_{m} v}{K}\right)}\right)\right] \\
& =1-e^{\frac{j 2 \pi k_{l} v}{K}} E\left[e^{j \phi_{k_{l}}}\right]-e^{-j 2 \pi k_{m} v / K} E\left[e^{-j \phi_{k_{m}}}\right] \\
& +e^{\frac{j 2 \pi\left(k_{l}-k_{m}\right) v}{K}} E\left[e^{j\left(\phi_{k_{l}}-\phi_{k_{m}}\right)}\right] \\
& =1-e^{\frac{j 2 \pi k_{l} v}{K}} e^{\frac{-k_{l} \sigma_{p}^{2}}{2}}-e^{\frac{-j 2 \pi k_{m} v}{K}} e^{\frac{-k_{m} \sigma_{p}^{2}}{2}} \\
& +e^{\frac{j 2 \pi\left(k_{l}-k_{m}\right) v}{K}} e^{\frac{-\left|k_{l}-k_{m}\right| \sigma_{p}^{2}}{2}} .
\end{aligned}
$$

The best training signal depends on values of $v, \sigma_{p}^{2}$ and $\left\{\sigma_{m}^{2}\right\}$ and it will not remain optimal for all possible values of $v$ and $\sigma_{p}^{2}$. Furthermore, a closed-form analytical solution to the above training design appears to be intractable. Hence, we adopt the following approach. Define

$$
\begin{aligned}
\boldsymbol{B}(k) & =\left[\left|A_{k, 0}\right|^{2}, \ldots,\left|A_{k, d_{k}-1}\right|^{2}\right]^{T} \\
\overline{\boldsymbol{V}}(m, k) & =\left[\bar{V}_{m+l_{k, 0}}, \ldots, \bar{V}_{m+l_{k, d_{k}-1}}\right]^{T} \\
\boldsymbol{\Omega}(k) & =\sum_{m=0}^{L-1} \sigma_{m}^{2} E\left[\overline{\boldsymbol{V}}(m, k) \overline{\boldsymbol{V}}^{H}(m, k)\right] \\
\boldsymbol{B} & =\left[\boldsymbol{B}^{T}(0), \ldots, \boldsymbol{B}^{T}\left(N_{\mathrm{Tx}}-1\right)\right]^{T} \\
\boldsymbol{\Omega} & =\operatorname{diag}\left\{\boldsymbol{\Omega}(0), \ldots, \boldsymbol{\Omega}\left(N_{\mathrm{Tx}}-1\right)\right\} .
\end{aligned}
$$

Then, the objective function to minimize can be expressed as

$$
\operatorname{Tr}\left[\overline{\boldsymbol{X}}_{d}\right]=\boldsymbol{B}^{T} \boldsymbol{\Omega} \boldsymbol{B} .
$$

First, we suppress contribution from the off-diagonal elements of $\Omega$ by considering a diagonal matrix $\Omega$ which is obtained by using $d_{k}=1$ (and hence, $\left|A_{k}\right|^{2}=E_{\mathrm{av}}$ ). Then, we have

$$
\begin{aligned}
\operatorname{Tr}\left[\overline{\boldsymbol{X}}_{d}\right] & =\sum_{k=0}^{N_{\mathrm{Tx}}-1}\left|A_{k, 0}\right|^{4} \sum_{m=0}^{L-1} \sigma_{m}^{2} E\left[\left|\bar{V}_{m+l_{k}}\right|^{2}\right] \\
& =E_{\mathrm{av}}^{2} \sum_{k=0}^{N_{\mathrm{Tx}}-1} \sum_{m=0}^{L-1} \sigma_{m}^{2} E\left[\left|\bar{V}_{m+l_{k}}\right|^{2}\right] .
\end{aligned}
$$

Second, assuming a small frequency offset ${ }^{1}$, we have

$$
E\left[\left|\bar{V}_{l}\right|^{2}\right]=2\left[1-e^{\frac{-k_{l} \sigma_{p}^{2}}{2}} \cos \left(2 \pi k_{l} v / K\right)\right] \simeq 2\left(1-e^{-k_{l} \sigma_{p}^{2} / 2}\right) .
$$

\footnotetext{
${ }^{1}$ After frequency offset estimation and compensation, the residual frequency offset just before the channel estimation will be definitely small.
}

By substituting (31) into (30), the training design becomes

$$
\left\{l_{k}\right\}^{*}=\underset{\left\{l_{k}\right\}}{\arg \min } \sum_{k=0}^{N_{\mathrm{Tx}_{x}}-1}\left(1-e^{-l_{k} \sigma_{p}^{2} / 2}\left(\sum_{m=0}^{L-1} \sigma_{m}^{2} e^{-m \sigma_{p}^{2} / 2}\right)\right)
$$

where $l_{k} \neq l_{m}$ if $k \neq m$, and $l_{m}-l_{k} \geq L, K+l_{k}-l_{m} \geq L$ for any $l_{m}>l_{k}$. The solution is easily obtained as

$$
\left\{l_{k}: k=0, \ldots, N_{\mathrm{Tx}}-1\right\}^{\star}=\left\{m L: m=0, \ldots, N_{\mathrm{Tx}}-1\right\} .
$$

The corresponding pilot tones are given by

$$
\begin{aligned}
& \left\{c_{k}[n]: k=0,1, \ldots, N_{\mathrm{Tx}}-1\right\}= \\
& \left\{\sqrt{E_{\mathrm{av}}} e^{j \varphi_{m}} e^{-j 2 \pi m n L / K}: m=0,1, \ldots, N_{\mathrm{Tx}}-1\right\}(34)
\end{aligned}
$$

where $\left\{\varphi_{m}\right\}$ are arbitrary phase factors. The above training signal design is one of the $\mathrm{CDM}(\mathrm{F})$ pilot allocation over all sub-carriers and is the same as the training signal design for $K>L N_{\mathrm{Tx}}$ in the presence of frequency-offset-only. The NMSE for the proposed training signal in the presence of frequency offset and phase noise is given by

$$
\begin{aligned}
\mathrm{NMSE}= & \frac{\sigma_{n}^{2}}{E_{\mathrm{av}}}+\frac{2}{L N_{\mathrm{Tx}}} \sum_{k=0}^{N_{\mathrm{Tx}}-1} \sum_{m=0}^{L-1} \\
& \sigma_{m}^{2}\left[1-e^{\frac{-(m+k L) \sigma_{p}^{2}}{2}} \cos (2 \pi(m+k L) v / K)\right]
\end{aligned}
$$

from which we can observe that larger $v$ and $\sigma_{p}^{2}$ result in a larger NMSE.

\section{Simulation Results and Discussions}

Simulation results on the performance comparison of several training signals in the presence of frequency offset ( $v=0.001$, $0.01,0.1$ ) and Wiener phase noise (with $\sigma_{p}^{2}=0.0001,0.0025$, 0.01 ) are presented in Figures 1 and 2 for $N_{\mathrm{Tx}}=1$ and 2, respectively. Multipath channels between pairs of transmit and receive antennas are assumed to be iid with $L=8$ uncorrelated Rayleigh fading taps and an exponential power delay profile having a $3 \mathrm{~dB}$ per tap decaying factor. In the figures, training\#1 represents our proposed training signal given in (34), training\#2 employs an FDM pilot allocation with $L$ tones for each antenna, training\#3 is of a $\operatorname{CDM}(\mathrm{F})$ allocation over $2 L$ sub-carriers, and training\#4 uses a $\mathrm{CDM}(\mathrm{F})$ allocation over all sub-carriers with a phase factor $2 \pi m n L_{1} / K,\left(L_{1}>L\right)$. Note that all training signals used are optimal in the absence of frequency offset and phase noise. The following remarks are in order:

1) The presence of phase noise makes our proposed training signals even more advantageous than in the presence of frequency offset only. As the phase noise variance increases, the NMSE difference between the proposed training signals and the reference training signals increases.

2) Phase noise with $\sigma_{p}^{2} \geq 0.0025$ introduces an NMSE floor.

3) For $K>L N_{\mathrm{Tx}}$ which is a more practical scenario, NMSE improvements of the proposed training signals are significant. A smaller $N_{\text {Tx }}$ gives a larger NMSE improvement of the proposed training signals. This trend is the same as the effect of frequency offset. 
In Figures 3-5, the effects of phase noise on the NMSE performance of the proposed training signal for $N_{\mathrm{Tx}}=1,2,8$ are presented for $v=0.001,0.01$, and 0.1 , respectively. The simulation results match with the theoretical NMSE result. The following observations are in order:

1) For SNR values of practical interest, the performance degradation due to phase noise with $\sigma_{p}^{2} \leq 0.0001$ is negligible.

2) Phase noise with a larger $\sigma_{p}^{2}$ introduces a larger NMSE floor.

3) Similar to the frequency offset effect, the performance degradation due to phase noise with a larger $\sigma_{p}^{2}$ becomes larger for a larger $N_{\mathrm{Tx}}$.

\section{CONCLusions}

We presented a training signal design for MIMO OFDM channel estimation in the presence of both frequency offset and phase noise. Frequency offset and phase noise introduce a channel estimation NMSE floor which is larger for larger frequency offset and phase noise level and a larger number of transmit antennas. The advantage of the proposed training signals over other training signals which are optimal in the absence of frequency offset and phase noise is greater for a smaller number of transmit antennas and a larger frequency offset and phase noise level. In the presence of both frequency offset and phase noise, the performance advantage of our proposed training signals over other training signals becomes even greater than in the case of frequency offset only.

\section{REFERENCES}

[1] H. Minn, V. K. Bhargava and K. B. Letaief, "A robust timing and frequency synchronization for OFDM systems," IEEE Trans. Wireless Commun., Vol. 2, No. 4, July 2003, pp. 822-839.

[2] H. Minn and S. Xing, "An optimal training signal structure for frequency offset estimation," IEEE Trans. Commun., Feb. 2005, pp. 343-355.

[3] H. Minn, S. Xing, and V. K. Bhargava, "Optimal periodic training signal for frequency offset estimation in frequency selective fading channels,"IEEE ICC'04, pp. 488-492, June 20-24, 2004.

[4] R. Negi and J. Cioffi, "Pilot tone selection for channel estimation in a mobile OFDM system," IEEE Trans. Consumer Electronics, Vol. 44, No. 3, pp. 1122-1128, Aug., 1998.

[5] J.H. Manton, "Optimal training sequences and pilot tones for OFDM systems," IEEE Commun. Letters, April 2001, pp. 151-153.

[6] S. Ohno and G. B. Giannakis, "Optimal training and redundant precoding for block transmissions with application to wireless OFDM" IEEE Trans. Commun., Vol. 50, No. 12, Dec. 2002, pp. 2113-2123.

[7] S. Adireddy, L. Tong, and H. Viswanathan, "Optimal placement of training for frequency-selective block-fading channels," IEEE Trans. Info. Theory, Vol. 48, No. 8, Aug. 2002, pp. 2338-2353.

[8] Y. Li, "Simplified channel estimation for OFDM systems with multiple transmit antennas," IEEE Trans. Wireless Commun., Jan. 2002, pp. 67-75.

[9] T.L. Tung, K. Yao, and R.E. Hudson, "Channel estimation and adaptive power allocation for performance and capacity improvement of multipleantenna OFDM systems," IEEE SPAWC, Mar. 2001, pp. 82-85.

[10] I. Barhumi, G. Leus, and M. Moonen, "Optimal training design for MIMO OFDM systems in mobile wireless channels," IEEE Trans. Signal Processing, Vol. 51, No. 6, June 2003, pp. 1615-1624.

[11] H. Minn and N. Al-Dhahir, "Optimal training signals for MIMO OFDM channel estimation," IEEE Globecom, Nov. 29-Dec. 3, 2004, pp. 219-224.

[12] H. Minn and N. Al-Dhahir, "Training signal design for MIMO OFDM channel estimation in the presence of frequency offsets," IEEE WCNC, March 13-17, 2005, Vol. 1, pp. 1-6.

[13] T. Pollet, M. Van Bladel and M. Moeneclaey, "BER sensitivity of OFDM systems to carrier frequency offset and Wiener phase noise," IEEE Trans. Commin., Vol. 43, No. 2/3/4, Feb/Mar/Apr 1995, pp. 191-193.

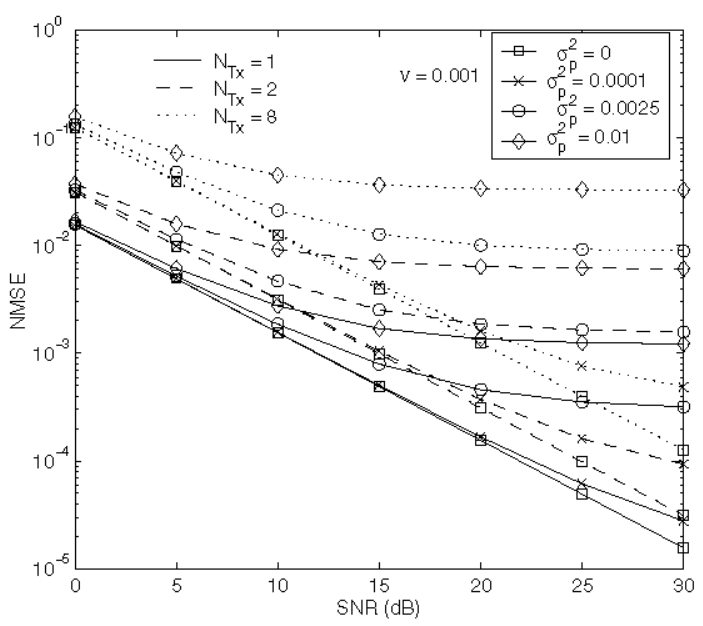

Fig. 3. The effect of phase noise on the NMSE of proposed training signal for a MIMO OFDM system with $K=64, N_{g}=16$, and $v=0.001$

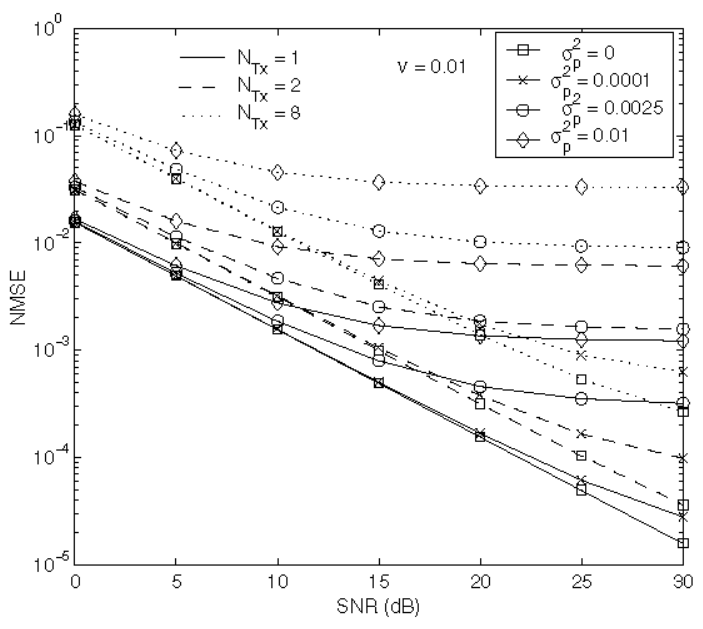

Fig. 4. The effect of phase noise on the NMSE of proposed training signal for a MIMO OFDM system with $K=64, N_{g}=16$, and $v=0.01$

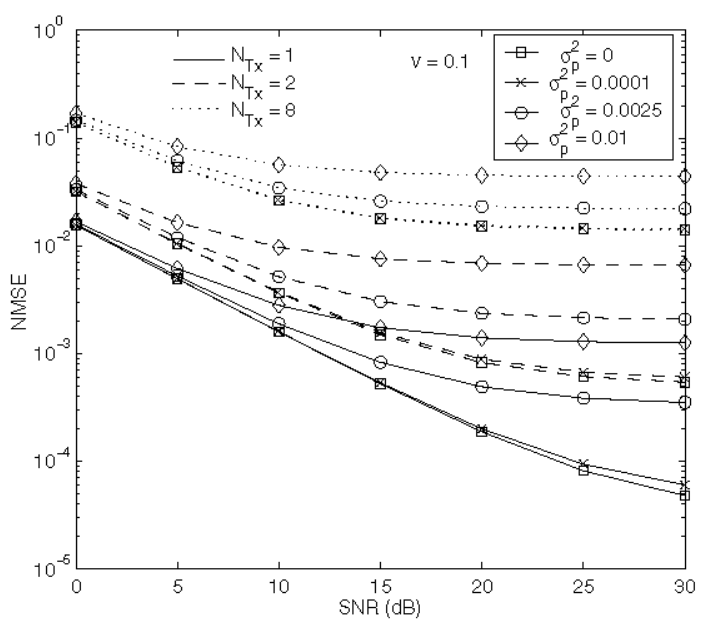

Fig. 5. The effect of phase noise on the NMSE of proposed training signal for a MIMO OFDM system with $K=64, N_{g}=16$, and $v=0.1$ 

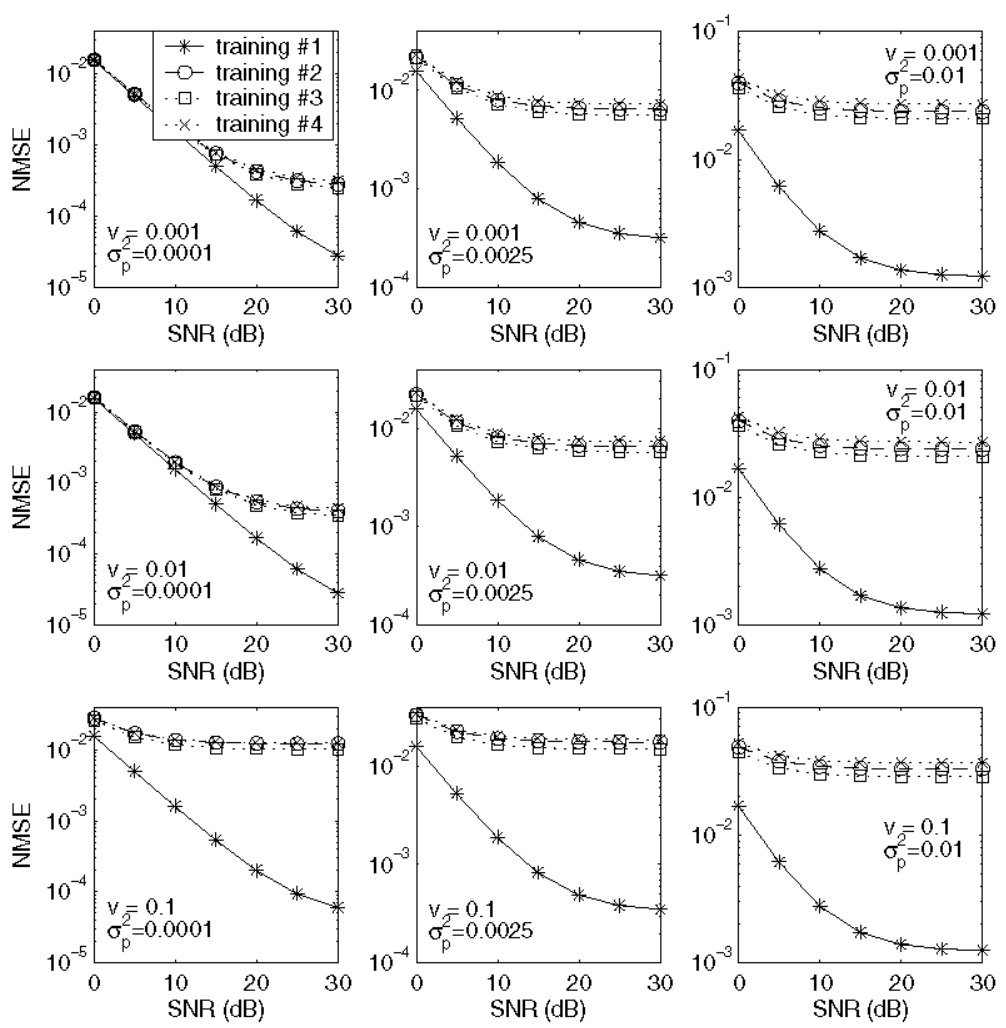

Fig. 1. The NMSE comparison of several training signals in the presence of frequency offset and phase noise for an $\mathrm{OFDM}$ system with $N_{\mathrm{Tx}}=1, K=64$, $N_{g}=16$
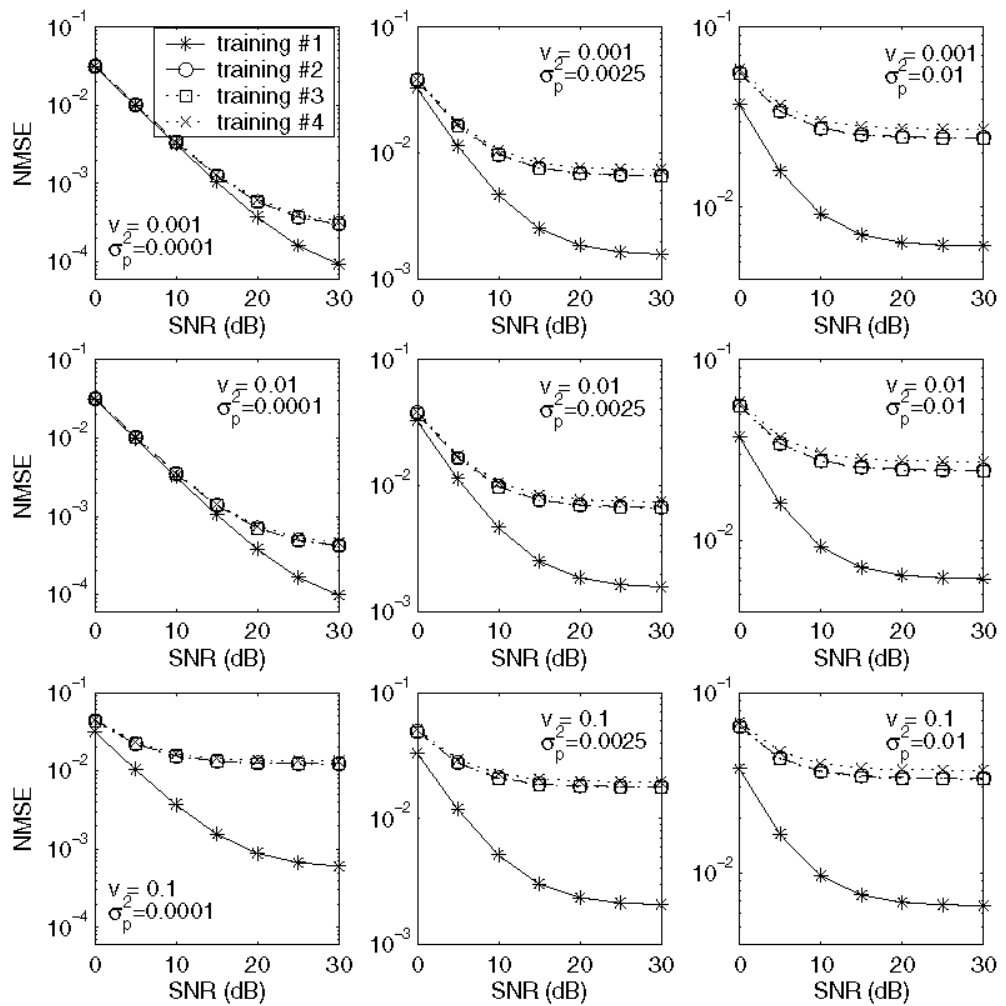

Fig. 2. The NMSE comparison of several training signals in the presence of frequency offset and phase noise for a MIMO OFDM system with $N_{\mathrm{Tx}}=2$, $K=64, N_{g}=16$ 